\title{
-NOTES-
}

\section{PURE BENDING AND TWISTING OF THIN SKEWED PLATES*}

\section{By ERIC REISSNER (Massachusetts Institute of Technology)}

1. Introduction. The following is concerned with the problems of pure bending and twisting in the theory of transverse bending of thin plates. Known results for rectangular plates of uniform thickness will be extended to skewed plates.

It was shown by Kelvin and Tait that the problem of St. Venant torsion of a thin rectangular plate with edges parallel to axes $x$ and $y$ has the solution $w=\theta x y$ where $w$ is the deflection function of the plate and $\theta$ the (constant) angle of twist per unit length. Within this theory, which neglects transverse shear deformation, the torque is applied to the plate by means of concentrated forces of suitable direction which act at the corners of the plate.

We will show here that Kelvin's and Tait's solution is readily extended to skewed plates of uniform thickness. In so doing we obtain, in particular, the influence of the angle of skew on the torque-twist relation for the plate. We also obtain a solution for the problem of pure bending of a skewed uniform plate. We find that pure bending of the skewed plate is associated with a twisting deformation the relative magnitude of which depends on the angle of skew.

2. Formulation of the problem. Let $x, y$ be mutually perpendicular directions in the undeflected middle surface of the plate. The differential equation for a uniform plate bent by edge forces and moments only is of the form

$$
\nabla^{2} \nabla^{2} w=0
$$

where $w$ is the deflection of the plate. Stress couples $M_{x}, M_{y}$ and $M_{x y}$, defined in the usual way, are

$$
\begin{aligned}
M_{x} & =-D\left(\partial^{2} w / \partial x^{2}+\nu \partial^{2} w / \partial y^{2}\right), \\
M_{\nu} & =-D\left(\partial^{2} w / \partial y^{2}+\nu \partial^{2} w / \partial x^{2}\right), \\
M_{x y} & =-(1-\nu) D \partial^{2} w / \partial x \partial y .
\end{aligned}
$$

For what follows we have no need for the corresponding expressions for the transverse stress resultants.

We consider plates bounded by two straight lines $x= \pm l$ and by two straight lines $y= \pm \frac{1}{2} c-x \tan \Lambda$. The angle $\Lambda$ is the angle of skew of the plate, $2 l$ is the span of the plate and $c$ is the chord of the plate.

Expressions for bending moment $M_{n}$ and twisting moment $M_{n t}$ acting along the edges $y= \pm \frac{1}{2} c-x \tan \Lambda$ follow by means of the usual transformation formulas for plate bending couples,

$$
\begin{aligned}
& M_{n}=M_{\nu} \cos ^{2} \Lambda+M_{x} \sin ^{2} \Lambda+2 M_{x \nu} \cos \Lambda \sin \Lambda, \\
& M_{n t}=\left(M_{x}-M_{\nu}\right) \cos \Lambda \sin \Lambda+M_{x \nu}\left(\cos ^{2} \Lambda-\sin ^{2} \Lambda\right) .
\end{aligned}
$$

Received February 18, 1952.

*The present paper is a report on work done under the sponsorship of the Office of Naval Research under Contract N5-ori-07834 with Massachusetts Institute of Technology. 
In addition to this we need Kelvin's and Tait's result that there occur concentrated forces $P$ at the corners of the plate given by

$$
P=\left(M_{x y}+M_{n t}\right)_{c \text { orner }}
$$

and that the effective transverse edge stress resultant $R_{n}$ is of the form $V_{n}+\partial M_{n t} / \partial s$.

3. Choice of deflection function. We shall find that for the problems of twisting and bending which are here considered it is sufficiently general to assume a deflection function

$$
w=\frac{1}{2} A x^{2}+\frac{1}{2} B y^{2}+C x y
$$

where $A, B$ and $C$ are constants. We then have a uniform distribution of stress couples

$$
M_{x}=-D(A+\nu B), \quad M_{\nu}=-D(B+\nu A), \quad M_{x \nu}=-(1-\nu) D C,
$$

vanishing transverse stress resultants $V_{x}$ and $V_{y}$, and vanishing effective edge stress resultants $R_{n}$.

4. Twisting of skewed plates. The following boundary conditions must be satisfied

$$
\begin{array}{ll}
x= \pm l ; & M_{x}=0 ; \\
y= \pm \frac{1}{2} c-x \tan \Lambda ; & M_{n}=0 .
\end{array}
$$

In addition to this we have that the applied torque $T$ is given by

$$
T=c P \text {. }
$$

Equations (7), (8) and (9) are three simultaneous equations for the determination of the three constants $A, B$ and $C$. We obtain

$$
C=-\frac{T}{2(1-\nu) c D}, \quad B=\frac{T \tan \Lambda}{\left(1-\nu^{2}\right) c D}, \quad A=-\nu \frac{T \tan \Lambda}{\left(1-\nu^{2}\right) c D}
$$

and therewith

$$
w=-\frac{T}{2(1-\nu) c D}\left[x y-\frac{\tan \Lambda}{1+\nu}\left(y^{2}-\nu x^{2}\right)\right] .
$$

The meaning of this result becomes somewhat more transparent if we introduce a new chordwise coordinate $\eta$ counted from the centerline $y=-x \tan \Lambda$ of the plate, by setting

$$
y=\eta-x \tan \Lambda .
$$

We then have

$$
w=-\frac{T}{2(1-\nu) c D}\left[\left(1+\frac{2 \tan ^{2} \Lambda}{1+\nu}\right) x \eta-\frac{\tan \Lambda}{1+\nu} \eta^{2}-\frac{\left(1+\tan ^{2} \Lambda\right) \tan \Lambda}{1+\nu} x^{2}\right] .
$$

We may define an effective angle of twist $\theta$ per unit length by means of the expression

$$
\theta=\frac{(w)_{\eta=c / 2}-(w)_{\eta=-r / 2}}{c x} .
$$

Combination of (13) and (14) gives the following result for this effective angle of twist

$$
\theta=-\frac{T}{2(1-\nu) c D}\left(1+\frac{2 \tan ^{2} \Lambda}{1+\nu}\right) .
$$


Equation (15) indicates that the skewed plate has a smaller torsional rigidity than the unskewed plate and in which way the torsional rigidity varies with the angle of skew $\Lambda$.

5. Pure bending of skewed plate. We denote the applied moment by $M$. The following boundary conditions must be satisfied

$$
\begin{array}{rlrl}
x & = \pm l, & c M_{x} & =M, \\
y & = \pm \frac{1}{2} c-x \tan \Lambda ; & M_{n}=0 .
\end{array}
$$

In addition to this we have the condition of vanishing torque, or of vanishing corner forces $P$, which becomes

$$
M_{x y}+M_{n t}=0 .
$$

From equations (16) to (18) we obtain the following expressions for the coefficients $A$, $B$ and $C$ in $w$

$$
A=-\frac{M}{\left(1-\nu^{2}\right) c D}, \quad B=\frac{\nu M}{\left(1-\nu^{2}\right) c D}, \quad C=\frac{M \tan \Lambda}{2(1-\nu) c D} .
$$

The deflection $w$ is now

$$
w=-\frac{M}{2\left(1-\nu^{2}\right) c D}\left[x^{2}-\nu y^{2}-(1+\nu) \tan \Lambda x y\right] .
$$

In terms of the chordwise variable $\eta$ defined by (12) this becomes

$$
w=-\frac{M}{\left(1-\nu^{2}\right) c D}\left[\left(1+\tan ^{2} \Lambda\right) x^{2}-(1-\nu) \tan \Lambda x \eta-\nu \eta^{2}\right] .
$$

We see from (21) that the skewed plate has a smaller bending stiffness than the unskewed plate and that moreover the applied bending moment produces also a torsional deformation.

\section{ON THE ERROR TERM IN INTERPOLATION FORMULAS}

\section{BY IVAN NIVEN (University of Oregon)}

From $n+1$ known values of a function $f(x)$ an approximating polynomial $p(x)$ of degree $n$ can be obtained. If $f(x)$ and its derivatives to order $n+1$ are continuous, then the error term can be calculated from

$$
f(x)-p(x)=\frac{f^{(n+1)}(\xi)}{(n+1) !} \prod_{i=0}^{n}\left(x-x_{i}\right),
$$

where $f\left(x_{i}\right)$ are the known values of the function for $j=0,1, \cdots, n, x$ is an arbitrary point in the interval $\left(x_{0}, x_{n}\right)$ with $x \neq x_{i}$ for every $j$, and $\xi$ is some point in the interval dependent on $x$. The purpose of this note is to indicate that no limit on the error can be obtained by replacing the term $f^{(n+1)}(\xi)$ by some function of an $n$-th difference $\Delta^{n+1} y$. (This replacement is made, for example, in Numerical Mathematical Analysis by J. B. 